\title{
ACUTE EFFECTS OF CUSTOMIZING A TENNIS RACKET ON SERVE SPEED
}

\author{
Mustafa Söğüt \\ Kırıkkale University, Kırlkkale, Turkey
}

\begin{abstract}
Background. A vast number of tennis players use additional lead weights attached to their rackets in order to improve stroke performances. However, there is limited evidence that racket customization as such boosts efficiency in different age and performance levels. The purpose of this repeated measures design study was to determine the acute effects of adding weight to the tip of a racket on serve speed in tennis players.

Methods. Participants were male tennis players from three different age and performance levels: elite junior $(n=$ 7 , age $=15.71 \pm 0.95$ years $)$, colligate $(n=11$, age $=22.36 \pm 2.54$ years $)$, and senior recreational $(n=17$, age $=52.94 \pm$ 9.43 years). Three identical rackets, two of which were customized by adding ten and twenty gram lead weights on the tips, were used for the measurements. A radar gun was utilized to assess serve speed of the participants.

Results. The repeated measures ANOVA results revealed no significant differences among serve speed scores for the three conditions in each group.

Conclusion. Results suggest that customizing a tennis racket by adding lead weight to the tip might not necessarily yield differences in serve speeds in tennis players from different age and performance levels.
\end{abstract}

Keywords: racket customization, serve speed, tennis.

\section{INTRODUCTION}

$\mathrm{C}$ ustomizing a tennis racket is defined as the modification on its certain points in order to render optimal feel and to enhance stroke performance (Cross, 2002). A vast majority of professional players generally customize their rackets by adding lead weights to the main strategic places such as the head or the handle (Brody, 1987; Cross, 2001, 2002; Cross \& Lindsey, 2005). These adjustments cause alternation in the weight, balance point (the location of racket's centre of mass), and swing weight (resistance of racket to rotation) of the racket (Cross, 2002; Lindsey \& Brody, 2002).

Adding lead weight specifically to the tip of a racket boosts swing weight (Lindsey \& Brody, 2002) and also yields improvement of racket power more than adding to any other particular location (Cross, 2001). This situation generates changes in the maximum power closer to the tip, and thus it provides additional height advantages for the server (Cross, 2001, 2002).
However, current literature presents limited evidence that might prove this advantage on serve speed. There is a paucity of information about the effects of adding weight on serve speed in tennis players from different age and performance levels. The purpose of the study was to determine the acute effects of adding weight to the tip of a racket on serve speed in elite junior, colligate and senior recreational tennis players. This information is to allow for further comparative and critical assessment of results for different groups of performance level and age.

\section{METHODS}

Participants. A total of thirty-five male tennis players from three separate groups (elite junior, colligate, and senior recreational) participated to the study. Table 1 presents the descriptive statistics about the anthropometric and training 


\begin{tabular}{|l|c|c|c|}
\hline \multicolumn{1}{|c|}{ Groups } & $\begin{array}{c}\text { Juniors } \\
(\boldsymbol{n}=7)\end{array}$ & $\begin{array}{c}\text { Colligates } \\
(\boldsymbol{n}=\mathbf{1 1})\end{array}$ & $\begin{array}{c}\text { Seniors } \\
(\boldsymbol{n}=\mathbf{1 7})\end{array}$ \\
\hline Age (year) & $15.71 \pm 0.95$ & $22.36 \pm 2.54$ & $52.94 \pm 9.43$ \\
\hline Height (m) & $1.81 \pm 0.07$ & $1.79 \pm 0.05$ & $1.76 \pm 0.03$ \\
\hline Weight $(\mathrm{kg})$ & $67.6 \pm 11.13$ & $72.9 \pm 6.24$ & $81.8 \pm 7.59$ \\
\hline BMI $\left(\mathrm{kg} / \mathrm{m}^{2}\right)$ & $20.46 \pm 2.06$ & $22.71 \pm 1.42$ & $26.31 \pm 2.40$ \\
\hline Experience (year) & $7.29 \pm 0.95$ & $8.91 \pm 4.23$ & $15.82 \pm 8.13$ \\
\hline Tennis practice (h/w) & $6.86 \pm 2.34$ & $5.55 \pm 2.34$ & $4.97 \pm 2.15$ \\
\hline Physical practice (h/w) & $2.71 \pm 1.25$ & $2.18 \pm 0.98$ & $2.41 \pm 2.35$ \\
\hline Total practice (h/w) & $9.57 \pm 3.05$ & $7.73 \pm 2.41$ & $7.38 \pm 3.13$ \\
\hline
\end{tabular}

Table 1. Means and standard deviations for anthropometric and training characteristics

characteristics of the participants. They were informed of the purpose of the study and the testing procedures. Informed consent forms were signed by the participants and by the parents of junior players.

Measures. Three identical rackets (strung weight $=290$ gram, head size $=632 \mathrm{~cm}^{2}$, string tension $=25 / 25.5$, string pattern $=16 \times 18$ ), two of which were customized by adding ten and twenty gram lead weights on the tips, were used. A radar gun (PR1000-BC; Ball Coach, Santa Rosa, Calif., USA) was utilized to assess the serve speeds.

Procedures. All measurements were performed in an indoor tennis court. Participants were initially asked to complete a standard warm up. They were, then, encouraged to serve with the highest speed from the deuce court randomly with each racket. Five successful serves with each racket were recorded and the means were used for the analysis.

Statistical Analysis. Descriptive statistics (mean $\pm S D$ ) were calculated for the variables. The Repeated Measures ANOVA was conducted to determine differences among performance scores for the three conditions in each group. Statistical significance level was set at $p<.05$.

\section{RESULTS}

The means and standard deviations for the serve speed scores of the participants are presented in Table 2. Results revealed no significant differences for three conditions in each group: juniors $(F(2$, $12)=2.82, p=.099)$, colligates $(F(2,20)=1.87$, $p=.180)$, seniors $(F(2,32)=1.31, p=.285)$.

\section{DISCUSSION}

The purpose of this repeated measures design study was to investigate the acute effects of adding ten and twenty gram lead weights to the tip of a racket on serve speed in tennis players from different age and performance levels. The results indicated no significant effects of additional weights on serve speed in each group. A supportive finding was observed by Whiteside, Elliott, Lay, and Reid (2014). They examined the effects of increasing a tennis racket's swing weight by $5 \%$ and $10 \%$ on

\begin{tabular}{|l|c|c|c|}
\hline Groups & Regular & 10 gram added & 20 gram added \\
\hline Junior & $156.53 \pm 8.66$ & $160.36 \pm 11.85$ & $158.70 \pm 9.27$ \\
\hline Colligate & $146.67 \pm 10.09$ & $149.40 \pm 9.71$ & $150.84 \pm 10.72$ \\
\hline Senior & $114.80 \pm 16.34$ & $115.74 \pm 17.22$ & $116.99 \pm 16.42$ \\
\hline
\end{tabular}

Table 2. Serve speed $\left(\mathbf{k m} \cdot \mathbf{h}^{-1}\right)$ scores (mean \pm standard deviation) for the three conditions 
serving arm kinematics and serve speed. Eleven elite junior female players were asked to serve with three rackets two of which were modified with lead tape. Their results showed that customizing swing weight by adding lead tape failed to boost acute increase in serve speed. It was noted that increment in the swing weight caused deceleration in the velocity of internal rotation in the serving arm which may explain standing data in serve speed. According to Elliott, Marshall, and Noffal (1995), velocity of the internal rotation during the serve is the main determinant factor (54.2\%) for the racket head speed.

Similar serve speed scores performed by the participants of the current study might be explained also with the mutual effect between swing speed and swing weight. In a recent paper, Cross and Bower (2006) reported the inverse interaction between swing weight and swing speed, namely when the swing weight increase the swing speed decrease. Similarly, Mitchell, Jones, and King (2000) studied the effects of racket inertia on head speed, and found significant increment in head speed when the swing weight decreases. According to Miller (2006) swing speed depends on the distribution of mass and when the mass is added to the tip of a racket this makes the swing harder.

It must be noted that this study is limited with influences of the ten and twenty gram of lead weight. Although Cross (2001) claimed that in a normal condition weights smaller than thirty grams are more convenient for the customization, a weight smaller than ten gram or heavier than twenty gram would yield significant increment in serve speed. Furthermore, one of the constraining factors of the study is regarding the rackets used in the measurements of serve speed. Instead of using their own rackets, participants were asked to use rackets provided by the researcher. Technical features such as grip size, string tension, head size, and balance point of the rackets used in the study might also be considered as restricting factors and might cause familiarization problems.

\section{CONCLUSION}

In conclusion, results of the study suggest that customizing a tennis racket by adding lead weight to the tip might not necessarily yield differences in serve speed in elite juniors, colligates, and senior recreational tennis players. Future studies that may focus on immediate and longitudinal effects of racket customization on both swing and serve speed in male and female professional tennis players are recommended.

Authors Note. This study was partly presented at the 8th National Biomechanics Congress in Ankara, Turkey.

\section{REFERENCES}

Brody, H. (1987). Tennis science for tennis players. Philadelphia, PA, USA: University of Pennsylvania Press.

Cross, R., \& Bower, R. (2006). Effects of swingweight on swing speed and racket power. Journal of Sports Sciences, 24(1), 23-30. doi: 10.1080/02640410500127876

Cross, R. (2001). Customising a tennis racket by adding weights. Sports Engineering, 4(1), 1-14.

Cross, R. (2002). Customizing racquets. In H. Brody, $\mathrm{R}$. Cross, and C. Lindsey (Eds.), The physics and technology of tennis (pp. 175-180). California: Usrsa.

Cross, R., \& Lindsey, C. (2005). Technical tennis: Racquets, strings, balls, courts, spin, and bounce. California: Racquet Tech Pub.

Elliott, B. C., Marshall, R. N., \& Noffal, G. J. (1995). Contributions of upper limb segment rotations during the power serve in tennis. Journal of Applied Biomechanics, 11, 433-442.

Lindsey, C., \& Brody, H. (2002). Weight, balance \& swing weight. In H. Brody, R. Cross, and C. Lindsey (Eds.), The physics and technology of tennis (pp. 2334). California: Usrsa.

Miller, S. (2006). Modern tennis rackets, balls, and surfaces. British Journal of Sports Medicine, 40(5), 401-405. doi: 10.1136/bjsm.2005.023283

Mitchell, S. R., Jones, R., \& King, M. (2000). Head speed vs. racket inertia in the tennis serve. Sports Engineering, 3(2), 99-110.

Whiteside, D., Elliott, B., Lay, B., \& Reid, M. (2014). The effect of racquet swing weight on serve kinematics in elite adolescent female tennis players. Journal of Science and Medicine in Sport, 17(1), 124-128. doi: 10.1016/j.jsams.2013.03.001 